\title{
Pathological game use in adults with and without Autism Spectrum Disorder
}

\author{
Christopher R Engelhardt ${ }^{1}$ ， Micah 0 Mazurek ${ }^{\text {Corresp., }}{ }^{2,3}$ ， Joseph Hilgard ${ }^{\text {Corresp. } 4}$ \\ ${ }^{1}$ CARFAX, Inc., Columbia, Missouri, United States \\ 2 Thompson Center for Autism and Neurodevelopmental Disorders, University of Missouri, Columbia, Missouri, United States \\ 3 Department of Health Psychology, University of Missouri, Columbia, Missouri, United States \\ 4 Annenberg Public Policy Center, University of Pennsylvania, Philadelphia, Pennsylvania, United States \\ Corresponding Authors: Micah O Mazurek, Joseph Hilgard \\ Email address: mazurekm@health.missouri.edu, jhilgard@gmail.com
}

This study tested whether adults with autism spectrum disorder (ASD) are at higher risk for pathological game use than typically developing (TD) adults. Participants included 119 adults with and without ASD. Participants completed measures assessing daily hours of video game use, percent of free time spent playing video games, and symptoms of pathological game use. The results indicated that adults with ASD endorsed more symptoms of video game pathology than did TD adults. This effect was strong, enjoying 300,000-to-1 odds in Bayesian model comparison. Results also showed that adults with ASD spent more daily hours playing video games and spent a higher percent of their free time playing video games than TD adults. Even after adjustment for these differences in daily video game hours and proportion of free time spent on games, model comparisons found evidence for a difference in game pathology scores associated with ASD status. Additionally, escapism motives for playing video games was associated with game pathology scores in both ASD and TD adults, replicating and extending a previous report. In conclusion, the risk for pathological game use appears larger in adults with ASD compared with TD adults. These findings point to pathological game use as a potentially important focus of clinical attention in adults with ASD. 
1

2

3

4

5

6

7

8

9

10

11

12

13

\title{
Pathological Game Use in Adults with and without Autism Spectrum Disorder
}

\author{
Christopher R. Engelhardt ${ }^{\mathrm{a}}$
}

Micah O. Mazurek ${ }^{b}$

Joseph Hilgard ${ }^{\mathrm{c}}$

${ }^{\mathrm{a} C A R F A X}$, Inc., Columbia, Missouri

Email: cre8f9@mail.missouri.edu

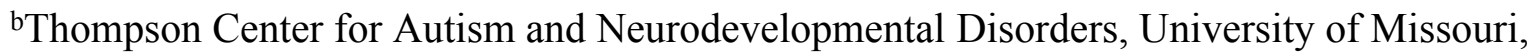
Columbia, Missouri; Department of Health Psychology, University of Missouri, Columbia, Missouri.

Email: mazurekm@missouri.edu

${ }^{\mathrm{c} A n n e n b e r g}$ Public Policy Center, University of Pennsylvania, Philadelphia, Pennsylvania Email: jhilgard@gmail.com

Please address correspondence to:

Micah O. Mazurek, $\mathrm{PhD}$

University of Missouri - Columbia

Columbia, MO

Phone: 573-884-5451

Fax: 573-884-6421

E-mail: mazurekm@missouri.edu 


\section{Abbreviations:}

\section{Abstract}

$51 \quad M=$ mean

RUNNING HEAD: Pathological Game Use in Adults with ASD

This study tested whether adults with autism spectrum disorder (ASD) are at higher risk for pathological game use than typically developing (TD) adults. Participants included 119 adults with and without ASD. Participants completed measures assessing daily hours of video game use, percent of free time spent playing video games, and symptoms of pathological game use. The results indicated that adults with ASD endorsed more symptoms of video game pathology than did TD adults. This effect was strong, enjoying 300,000-to-1 odds in Bayesian model comparison. Results also showed that adults with ASD spent more daily hours playing video games and spent a higher percent of their free time playing video games than TD adults. Even after adjustment for these differences in daily video game hours and proportion of free time spent on games, model comparisons found evidence for a difference in game pathology scores associated with ASD status. Additionally, escapism motives for playing video games was associated with game pathology scores in both ASD and TD adults, replicating and extending a previous report. In conclusion, the risk for pathological game use appears larger in adults with ASD compared with TD adults. These findings point to pathological game use as a potentially important focus of clinical attention in adults with ASD.

$\mathrm{ASD}=$ autism spectrum disorder

DSM = Diagnostic and Statistical Manual of Mental Disorders

$\mathrm{TD}=$ typically developing 
$52 S D=$ standard deviation 
53

54

55

56

57

58

59

60

61

62

63

64

65

66

67

68

69

70

71

72

73

74

75

\section{Introduction}

Autism spectrum disorder (ASD) is characterized by core deficits in social interaction/communication and by restricted interests and repetitive behaviors (American Psychiatric Association, 2013). These core impairments, which are often accompanied by cooccurring mental health problems such as depression and anxiety (Gillott \& Standen, 2007; Mazefsky, Folstein, \& Lainhart, 2008; Moseley, Tonge, Brereton, \& Einfeld, 2011), persist through adulthood (Seltzer, Shattuck, Abbeduto, \& Greenberg, 2004; Shattuck et al., 2007; Taylor \& Seltzer, 2010). As a result, adults with ASD have limited engagement in social and community activities and are at risk for poor independent living and employment outcomes (Eaves \& Ho, 2008; Howlin, Goode, Hutton, \& Rutter, 2004; Orsmond \& Kuo, 2011). Thus, identifying factors that affect health, well-being, and daily functioning in adults with ASD is of chief importance.

Among these factors, excessive video game play may be an important consideration.

Because social interactions are often hyper-stimulating or anxiety-provoking for individuals with ASD (Dalton et al., 2005; Joseph, Ehrman, McNally, \& Keehn, 2008), video games may be an especially appealing activity due to their predictability and low social demands. Consistent with this line of reasoning, previous studies have found that children and adolescents with ASD spent more than two hours per day playing video games. The time spent playing video games is greater than that of children with other disabilities (e.g., speech/language impairments, intellectual disabilities) or that of typically developing (TD) children (Mazurek \& Engelhardt, 2013b; Mazurek, Shattuck, Wagner, \& Cooper, 2012). Children with ASD also play more video games than do their TD siblings and spend far more time playing video games than on other extracurricular activities (Mazurek \& Wenstrup, 2013). Excessive play may be deleterious; 
76 problematic game use among children with ASD was associated with poor sleep and poorer

77 behavioral functioning (Engelhardt, Mazurek, \& Sohl, 2013; Mazurek \& Engelhardt, 2013a).

78

79

80

81

82

These findings are consistent with anecdotal reports from parents and clinicians that many individuals with ASD exhibit patterns of excessive video game play.

\subsection{Pathological video game use}

Pathological video game use is a significant clinical issue in its own right, included as "Internet Gaming Disorder", a "Condition for Further Study", in the DSM-5 (American Psychiatric Association, 2013). Although broad consensus has not been reached, many researchers view the construct as similar to other behavioral addictions such as gambling addiction (Griffiths, 2008; Griffiths, Kuss, \& King, 2012; Hellman, Schoenmakers, Nordstrom, \& van Holst, 2013; King, Haagsma, Delfabbro, Gradisar, \& Griffiths, 2013). Like gambling, video game play can be a source of enjoyment. However, video game play can become pathological if it leads to significant impairment or distress. Consistent with the definition of internet gaming disorder and gambling disorder in the DSM-5 (American Psychiatric Association, 2013), pathological game use is defined by its symptoms: preoccupation with video games, salience, euphoria/relief, tolerance, withdrawal symptoms, mood modification, conflict, relapse and reinstatement, unsuccessful attempts to control video game play, continued excessive use, deceiving others as to the amount of time spent gaming, and loss of interest in hobbies(American Psychiatric Association, 2013; King et al., 2013; Petry et al., 2014; Sim, Gentile, Bricolo, Serpelloni, \& Gulamoydeen, 2012). Previous research has found correlations between symptoms and amount of video game play, video game play to escape real life, selfperceptions of excessive game play, and scores on other measures of dependence (Gentile, 2009; Hart et al., 2009; Hilgard, Engelhardt, \& Bartholow, 2013; King, Delfabbro, \& Zajac, 2011; 
99 King et al., 2013; Lemmens, Valkenburg, \& Peter, 2009; Sim et al., 2012; Tejeiro Salguero \&

100 Morán, 2002). This research supports pathological game use as a reliable and valid construct

101 (but see Scharkow, Festl, and Quandt (2014) for concerns about test-retest stability and

102 reliability).

103 Pathological game use is a clinically relevant problem in the general population. Adults

104 who play video games to excess have less time for other activities, including work, educational

105 pursuits, hobbies, socialization with friends and family members, and sleep (Eickhoff et al.,

106 2015; Griffiths, Davies, \& Chappell, 2003). In addition, pathological video game use is

107 associated with a number of negative psychosocial indicators, including aggression (Griffiths \&

108 Hunt, 1998; Grüsser, Thalemann, \& Griffiths, 2006), depression (Feng et al., 2003; Gentile et al.,

109 2011), anxiety (Gentile et al., 2011), poor life satisfaction (Lemmens, Valkenburg, \& Peter,

110 2011; Mentzoni et al., 2011), loneliness (Lemmens et al., 2009), and interference with social

111 relationships (Lemmens et al., 2011; Smyth, 2007). Negative health effects also include reduced

112 sleep (Smyth, 2007), risk of game-induced seizures (Chuang, 2006), and poor physical health

113 (Kelley \& Gruber, 2012). Overall, both researchers and clinicians have concluded that the real-

114 world problems associated with pathological game use are worthy of future investigation.

The well-being of adults with ASD could benefit from attention to potential symptoms of

116 pathological game use. Individuals with ASD are at risk for social isolation, preoccupation with

117 specific interests, and repetitive patterns of behavior (American Psychiatric Association, 2013).

118 As such, individuals with ASD may be at particular risk for excessive use of video games.

119 Consistent with this idea, prior studies have found that individuals with ASD may have a

120 complicated relationship with video games. On the one hand, qualitative findings suggest that

121 adults with ASD report a number of positive aspects of video game use, including stress 
122 reduction and social connection (Mazurek, Engelhardt, \& Clark, 2015). On the other hand,

123 children and adolescents with ASD have difficulty disengaging from video games (MacMullin,

124 Lunsky, \& Weiss, 2015; Mazurek \& Engelhardt, 2013b; Mazurek \& Wenstrup, 2013). For

125 example, research has shown that boys and girls with ASD endorsed more symptoms of

126 pathological game use than did their TD siblings (Mazurek \& Wenstrup, 2013). This group

127 difference was replicated in a sample of unrelated boys with and without ASD, finding a large $(d$

$128>1$ ) difference between groups (Mazurek \& Engelhardt, 2013b). Results such as these indicate a

129 clinically meaningful difference in pathological game use in individuals with and without ASD.

130 Although previous research has documented differences in pathological game use in

131 children and adolescents with and without ASD, no study has examined whether adults with

132 ASD are at higher risk for pathological game use than TD adults. This is a particularly important

133 research topic because adults with ASD often suffer from poor social outcomes (Billstedt,

134 Gillberg, \& Gillberg, 2005; Billstedt, Gillberg, \& Gillberg, 2007; Howlin et al., 2004; Levy \&

135 Perry, 2011; Müller, Schuler, \& Yates, 2008). As adults have greater responsibilities and

136 autonomy than children, preoccupation with video games in individuals with ASD may be more

137 harmful to functioning among adults than among children. Difficulty disengaging from video

138 games may prevent adults from engaging in productive employment, education, or community

139 activities.

140 Consistent with this idea, recent qualitative research has shown that adults with ASD

141 express concerns about their preoccupation with video games. In this research, 10-15\% of

142 participants reported playing video games out of compulsion or routine and voiced concerns

143 about feeling addicted to games. Many also expressed that they played games in order to fill time

144 and that they often played games to the exclusion of other activities (Mazurek et al., 2015). Thus, 
145 there is reason to suspect that adults with ASD might be at greater risk for pathological game use

146 than adults without ASD.

\section{$147 \quad 1.2 \quad$ Current study}

148 The goal of the current study was to examine differences in video game play in adults

149 with and without ASD. The primary prediction was that adults with ASD would be at higher risk

150 for pathological game use than TD adults. We also predicted that adults with ASD would play

151 more video games per day and spend a higher percent of their free time playing video games

152 than TD adults. We tested these predictions using a model comparison approach with Bayes

153 factors, enabling us to directly examine the amount of evidence in favor of or against a group

154 difference in pathological game use.

155

156

157

158

159

160

161

162

163

164

165

166

167

\section{Methods}

This manuscript represents a secondary analysis of a dataset collected in a previous report (Engelhardt, Mazurek, Hilgard, Rouder, \& Bartholow, 2015). In that publication, we reported the results of an experiment in which ASD and TD individuals were randomly assigned to play a violent or nonviolent game; the effects of game violence on aggressive thoughts, feelings, and behaviors were described. In this publication, we describe correlations in questionnaire data collected alongside that experiment. A more comprehensive description of participant demographics, eligibility criteria, recruitment, and study procedure can be found in the original report. approval number \#1206273). All participants provided written consent prior to participating in the study. Participants received \$20 in exchange for their participation. Data and materials are available at https://osf.io/5dsnc/. 
$168 \quad 2.1 \quad$ Participants

169 Participants included 119 adults (16 women), half of whom had a previous diagnosis of

170 ASD, participating in a larger study on violent video game effects (Engelhardt et al., 2015). One

171 individual with ASD participated in the primary study but elected to withdraw from it prior to

172 completing the measures in this cross-sectional study. The sample was primarily White ( $85 \%$ of

173 the ASD group, $93 \%$ of the TD group) and non-Hispanic (85\% of the ASD group, $100 \%$ of the

174 TD group).

175 Participants in the ASD group were recruited through an academic medical center

176 specializing in ASD diagnosis and treatment. These participants had a previous diagnosis of

177 ASD based on the center's clinical care model. The diagnostic process generally includes

178 structured interviews, behavioral observation, and evaluations conducted by psychologists and/or

179 physicians using standardized assessment tools such as the Autism Diagnostic Observation

180 Schedule (ADOS) (Lord, DiLavorne, \& Risi, 2002) and/or the Autism Diagnostic Interview -

181 Revised (ADI-R) (Lord, Rutter, \& Le Couteur, 1994). All participants in the ASD group were

182 verbally fluent and high-functioning, with IQ scores greater than or equal to 85 . Most

183 participants in the ASD group were attending school (39\% full-time and 20\% part-time). The

184 majority (64\%) of participants in the ASD group were not currently employed, although 3\% had

185 full-time jobs and $32 \%$ had part-time jobs.

186 Participants in the TD group were recruited through the community and the university

187 campus using email, campus flyers, and face-to-face recruitment. Participants in the TD group

188 had no history of neurological or developmental disorders. Most participants in the TD group

189 were also attending school (95\% full-time and 3\% part-time), and most were also currently

190 employed (3\% full-time and 65\% part-time). The ASD and TD groups did not differ in age, 
191 gender, or IQ. Demographics are supplied in Table 1. Further details are available in Engelhardt

192 et al. (2015)

$193 \quad 2.2 \quad$ Measures

194 2.2.1 Video Game Use. Participants reported the number of hours over the past year

195 they spent playing video games on a typical weekday and weekend day. These were highly

196 correlated ( $\mathrm{r} \geq .85$ in both groups), so they were combined to make average time spent playing

197 video games per day, calculated by multiplying the weekday value by 5 and the weekend value

198 by 2 , summing these scores, then dividing by 7 , consistent with previous research (Orsmond \&

199 Kuo, 2011). Participants also indicated the percent of their free time spent playing video games.

200 At the end of the study, the researcher asked "What percent of your free time do you spend

201 playing video games?" If the respondent answered with a range of values, the midpoint was used

202 for analysis (e.g., "15-20\%" was coded as 17.5\%). Although we expect these two variables to be

203 positively correlated, we do not consider them as the same construct: depending on one's

204 obligations, a 1-hour game session could occupy a little of one's free time or all of it.

205 2.2.2 Pathological Game Use. Participants completed a 10-item measure of

206 pathological video game use adapted from previous research (Gentile, 2009; Hilgard et al.,

207 2013). The measure was originally developed to be consistent with DSM-IV criteria for another

208 behavioral addiction (pathological gambling), and items shared features with widely-used

209 definitions of addiction, including salience, euphoria or relief, tolerance, withdrawal, conflict,

210 relapse and reinstatement (see for detail Gentile, 2009). For example, the measure includes

211 questions about withdrawal ("In the past year, have you become restless or irritable when

212 attempting to cut down or stop playing video games?"), conflict with health and hygiene ("In the

213 past year, have you ever skipped sleeping, eating, or bathing so that you could spend more time 
214 playing video games?"), preoccupation (“In the past year, have you become more preoccupied

215 with playing video games, studying video game playing, or planning the next chance to play?"),

216 tolerance ("In the past year, have you needed to spend more and more time on video games to

217 achieve the same level of excitement?"), and deceit ("In the past year, have you ever lied to

218 family or friends about how much you play video games?"). Participants indicated whether they

219 had experienced each symptom over the previous year by responding "Yes," "Sometimes," or

220 "No," scored as 1, 0.5, and 0 points, respectively (Gentile, 2009; Hilgard et al., 2013). The scale

221 has demonstrated good convergent and divergent construct validity in prior research (Gentile,

222 2009). Cronbach's alpha coefficients were used to evaluate internal consistency of the scale in

223 the current sample. Item reliability estimates were good, with Cronbach's alpha of .788 for the

224 scale for the entire sample, $\alpha=.720$ for the ASD group, and $\alpha=.810$ for the TD group.

225 2.2.3 Escapism motives. As a secondary analysis, we hoped to replicate the relationship

226 between escapism motives for game use and symptoms of PGU we had found in an earlier report

227 (Hilgard et al., 2013). Participants rated each item from the GAMES escapism subscale on a 1-5

228 scale (Hilgard et al., 2013).

\section{3. Results}

\subsection{Analytic approach}

In response to numerous critiques levied against null hypothesis significance tests and

95\% confidence intervals, we used Bayes factors to state evidence for or against our predictions.

Bayes factors are reported in ratios such as 4-to-1 in favor of or against statistical models. These

evidence ratios can be interpreted as the probability of the observed data under one model compared with the probability of the observed data under a different model. They can also be interpreted as how beliefs should be updated in light of data. Computations were conducted in 
237 the BayesFactor package for R (Morey, Rouder, \& Jamil, 2014) using the GeneralTestBF

238 function and 100,000 Monte Carlo iterations.

239 We examined evidence for all possible model combinations. This strategy enabled us to

240 determine the best-fitting model and the amount of evidence in favor of or against a parameter in

241 that model. For example, in the analysis in which game pathology scores were included as the

242 dependent variable and group diagnosis, average time spent playing video games per day, and

243 the percent of free time spent playing video games were included as the independent variables,

244 seven models were compared: the null effects model, the model with group diagnosis only, the

245 model with average time spent playing video games per day only, the model with percent of free

246 time spent playing video games only, three models including two of the three parameters, and a

247 full model including all three parameters.

248 The models presented below used a JZS default prior. This Cauchy-distributed prior has

249 spread to cover reasonable effect sizes, specifies that smaller effects are more likely than larger

250 effects, and is centered at 0 . Consistent with previous research showing that boys with ASD

251 experience more problems with problematic video game use compared with TD boys (Mazurek

252 \& Engelhardt, 2013b), we tuned the scale parameter for the prior on the effects of both

253 categorical and continuous predictors to 1.12 , a large effect. This prior assigns $50 \%$ of

254 probability to effects $|\delta|=1.12$ or smaller. The remaining probability is assigned to larger effect 255 sizes.

256 We used general linear models to test our predictions. Because visual inspection of the 257 pathological game use scores indicated positive skew (Figure 1), a Box-Cox transformation was 258 applied to this outcome. The suggested transformation, raising game-pathology scores to the -.26 
259 power, helped normalize the residuals. We analyzed this transformed variable in the general

260 linear models presented below.

$261 \quad 3.2 \quad$ Symptoms of pathological game use

262 Our primary prediction was that adults with ASD would exhibit higher pathological game

263

264

265

266

267

268

269

270

271

272

273

274

275

276

277

278

279

280

281

use scores than TD adults. Adults with ASD indicated more symptoms than did TD adults

(untransformed $M(S D)=2.46(1.77)$ and 1.20 (1.55) for the ASD and TD groups, respectively, $d$ $=0.84[0.47,1.22], t(117)=4.61$. Bayesian analysis indicated that the observed difference of transformed values was 300,000 times more likely to reflect a hypothesized difference than no difference.

We next explored which features served to predict pathological game use scores through the comparison of multiple regression models. These models could include as predictors each of group diagnosis, average time spent playing video games per day, and/or the percent of free time spent playing video games. This lead to seven candidate models, representing the inclusion of one, two, or all three additive effects. All seven models were preferred to the null effects model. Bayes factor model comparisons suggested that the best-fitting model included parameters for diagnostic group $(b=0.22)$ and for the percent of free time spent playing video games $(b=0.01)$. This model was strongly preferred to the null effects model by a factor of $6.4 \times 10^{11}$ (or in frequentist terms, $F(2,116)=42.1)$.

By dropping individual terms from this model, one can assess the evidence for each effect conditional on adjustment for the other effects. For example, we dropped the effect of autism group from the above model, comparing the change in model fit with and without this effect. This model comparison yielded a Bayes factor of 3.0-to-1 $(t(116)=2.61)$ in favor of an effect for diagnostic group, even after adjustment for the effect of percentage of free time spent 
282 playing video games. Similar model comparisons yielded a Bayes factor of more than 1,400,000-

283 to-1 $(t(116)=6.18)$ in favor of an effect for the percent of free time spent playing video games,

284 even after adjustment for the effect of diagnostic group.

285 These regression models were then performed separately within each group to explore

286 how their influence might differ between ASD and TD adults. Scatterplots are provided in Figure

287 2. Within the ASD group, the best-fitting model modeled symptoms as a function of the

288 proportion of free time, $F(1,57)=13.5, \mathrm{BF}=37, b=0.01$. Average daily game play did not

289 strongly predict symptoms whether adjusting for $(\mathrm{BF}=0.14$, or 7.3 -to-1 against, $b=0.01, t(56)$

$290=0.26)$ or not adjusting for $(\mathrm{BF}=0.58$, or 1.71 -to- 1 against, $b=0.05, t(57)=1.97)$ the

291 proportion of free time spent playing games. Within the TD group, the best-fitting model

292 modeled symptoms as a function of the additive effects of both proportion of free time $(b=0.01$,

$293 t(57)=2.84)$ and daily hours spent playing video games $(b=0.17, t(57)=3.07), \mathrm{BF}=1.3 \times 10^{7}$,

$294 F(2,57)=30.75$. For this group, average daily game play did provide additional prediction of

295 symptoms, even when adjusting for the proportion of free time spent playing games, $\mathrm{BF}=4.73$.

\section{$296 \quad 3.3 \quad$ Tests of second prediction}

297 Zero-order correlations between all study variables can be seen in Table 2. Adults with

298

299

300

301

302

303

304

ASD spent more time playing video games per day $(M=2.80 \mathrm{~h}, S D=2.14)$ and spent a greater percent of their free time playing video games $(M=41.02, S D=27.38)$ than did TD adults $(M s=$ $1.04 \mathrm{~h}(1.20)$ and 14.25 (15.94), respectively). These effects were large in magnitude, $d=1.01$ $[0.63,1.40], t(117)=5.53$, and $1.20[0.80,1.59], t(117)=6.53$. Bayes factors found strong evidence of group differences in both of these variables: in each analysis, the model with group diagnosis was preferred to the null effects model by more than a factor of 59,000-to- 1 . Table 2 also shows that pathological game use symptoms are highly and positively associated with both 
305

306

307

308

309

310

311

312

313

314

315

316

317

318

319

320

321

322

323

324

325

326

327

average time spent playing video games per day and with the percent of free time spent playing video games, consistent with a number of previous studies. Table 3 presents the bivariate correlations for TD and ASD groups separately.

3.3.1 Replication of Hilgard et al. (2013). In a previous study, Hilgard et al. (2013)

developed a questionnaire measure of motives for playing video games. In that report, they found a positive relationship between playing games to escape real-life problems and symptoms of PGU. We replicated this relationship in both the ASD $(b=0.21, \mathrm{BF}=19.9, t(57)=3.29)$ and TD $(b=0.25, \mathrm{BF}=265, t(58)=4.24)$ groups. Additionally, escapism scores were significantly higher in the ASD group as compared to the TD group, $d=0.81[0.43,1.19], \mathrm{BF}=846, t(117)=$ 4.42 .

\section{Discussion}

This is the first study to examine pathological game use in adults with and without ASD. Consistent with our primary prediction, we found very strong evidence that adults with ASD are at higher risk for pathological game use symptoms than TD adults. Evidence for this group difference remained even after modeling the average amount of time spent playing video games per day and the percent of free time spent playing video games. Consistent with our second prediction, we found very strong evidence that adults with ASD both spent more time playing video games per day and spent a greater percentage of their free time playing video games than TD adults. These findings are consistent with previous research among children with ASD (Mazurek and Engelhardt, 2013b; Mazurek and Wenstrup, 2013), suggesting that the greater risk for pathological game use may persist into adulthood for many individuals with ASD. Additionally, some individual differences were found to correlate with PGU symptom counts. Among the TD group, both greater hours per day and greater proportion of free time 
328 spent gaming were associated with greater symptom counts. Among the ASD group, however,

329 only proportion of free time spent gaming had a strong correlation with symptom counts.

330 Relationships between these measures and symptom counts were weaker in the ASD group than

331 in the TD group; whether this is because the ASD group has greater difficulty with self-report or

332 less regimented use of time is unclear. Still, both groups showed a relationship between escapism

333 motives and PGU symptoms, replicating and extending the result described in Hilgard et al., 3342013.

The results here suggest that pathological game use may be an important clinical consideration for this population. Adults with ASD may be at high risk for problematic game use due to particular aspects of the behavioral phenotype of ASD. For example, highly restricted interests, preoccupations, and perseverative interests are key diagnostic features of ASD manifest as preoccupations with video games or compulsive patterns of game-play. Problematic game-play patterns may themselves have additional deleterious effects on overall functioning. Adults with ASD are already at risk for poor outcomes, including reduced engagement in social and community activities, education, and employment (Eaves \& Ho, 2008; Howlin et al., 2004; video games may exacerbate these difficulties, displacing time that could be spent on social, occupational, or other recreational activities (Mazurek et al., 2015; Mazurek \& Wenstrup, 2013).

347 Furthermore, given evidence from the general population, pathological game use may exacerbate core or co-occurring symptoms among adults with ASD, particularly worsening mood, anxiety, irritability, and social isolation (Feng et al., 2003; Gentile et al., 2011; Griffiths \& Hunt, 1998;

350 Grüsser et al., 2006; Lemmens et al., 2011; Mentzoni et al., 2011; Smyth, 2007). Additional 
351 research is needed to examine the specific psychosocial consequences of pathological game use

352 in adults with ASD. This information will be critical for informing effective interventions for

353 this problem.

\section{$354 \quad 4.1 \quad$ Limitations and Future Directions}

355 These data are cross-sectional and do not permit a causal interpretation of the current

356

357

358

359

360

361

362

363

364

results. Longitudinal and experimental studies are necessary to determine both the predictors, mediators, and outcomes of pathological game use among individuals with ASD. Additionally, we did not measure the absolute amount of free time participants had. Individuals with ASD may have fewer responsibilities (e.g., school, employment) than TD peers, which may account in part for their greater use of video games. Whether pathological game use is a cause or consequence of these reduced responsibilities is beyond the scope of this study, but it may be interesting to research in longitudinal designs.

Another potential limitation of the study is its reliance on self-report measurements. This strategy could be problematic because previous studies have suggested that individuals with ASD have difficulty understanding and reporting on their own experiences (Ben Shalom et al., 2006; Losh \& Capps, 2006). This may be why relationships between time spent playing games and PGU symptoms were weaker in the ASD group than the TD group. However, in order to enhance the validity of responses, participants were given an opportunity to ask the experimenter for clarification if survey questions seemed confusing. Participants were also pre-screened to ensure that they were fluent in English, could read and write on their own, and had an estimated IQ score greater than 85 prior to study enrollment.

Another potential limitation of the current study is that the measure of pathological video game use was relatively brief and covered a very wide time span (i.e., the past year). Although 
374 this measure has demonstrated good construct validity in prior research (Gentile, 2009), future

375 work on this topic may benefit from further development of more comprehensive measures

376 focused on a shorter recall period to enhance reliability and validity of responses.

377 Although the current study focused primarily on pathological aspects of video game use,

378 there is also room for research into the potential positive aspects of game use among adults with

379 ASD. Parents, researchers, and practitioners are interested in the potential of games for

380 improving social and behavioral functioning in children with ASD (Durkin, Boyle, Hunter, \&

381 Conti-Ramsden, 2015; Ferguson, Anderson-Hanley, Mazurek, Parsons, \& Warren, 2012;

382 Grynszpan, Weiss, Perez-Diaz, \& Gal, 2014; Moore \& Taylor, 2000). Expanding this work to

383 include a focus on improving outcomes for adults with ASD would be beneficial. Overall, more

384 research is needed to inform the development of interventions focused both on positive

385 applications of game-based technology and on reduction of potentially negative effects of

386 pathological game use.

$387 \quad 4.2 \quad$ Conclusions

These results suggest that adults with ASD may be at risk for pathological game use.

389 Future research is needed to identify the prevalence, course, predictors, and outcomes of video

390 game pathology in individuals with ASD. The current findings suggest that clinicians should

391 consider assessing these potential problems in clients. It may be important to consider the

392 potential impact of video game use on emotional, behavioral, and social outcomes among adults 393 with ASD.

394

395 
396 Table 1. Demographic Characteristics of the Two Diagnostic Groups

\begin{tabular}{lrrrrrrr} 
& & & & & \multicolumn{3}{c}{ Daily gameplay (hrs) } \\
\cline { 6 - 7 } Group & Male(\%) & Age & ABIQ & AQ & Weekday & Weekend & Escapism \\
ASD & 86 & 20.42 & 103.1 & 70.24 & 2.49 & 3.57 & 3.61 \\
TD & 87 & 20.54 & 103.4 & 57.17 & 0.83 & 1.56 & 2.89
\end{tabular}

397

398 Note: $\mathrm{ABIQ}=$ Abbreviated Battery IQ. AQ = Autism Short Questionnaire. Escapism = Escapism 399 subscale score from GAMES questionnaire. 
400 Table 2. Bivariate Relationships among all Study Variables

$\begin{array}{lrrrrrrr} & \text { Group } & \text { Gender } & \text { Hours } & \text { FreeTime\% } & \text { Escapism } & \text { RawPGU } & \text { PGU } \\ \text { Group } & 1.00 & & & & & & \\ \text { Gender } & .00 & 1.00 & & & & & \\ \text { Daily game use (hrs) } & .46 & .11 & 1.00 & & & \\ \text { Free time gaming (\%) } & .52 & .26 & .67 & 1.00 & & \\ \text { GAMES Escapism } & .38 & .32 & .33 & .52 & 1.00 & \\ \text { PGU symptoms (raw) } & .39 & .27 & .44 & .59 & .47 & 1.00 & \\ \text { PGU symptoms } & .48 & .31 & .53 & .62 & .55 & .94 & 1.00 \\ \text { (transformed) } & & & & & & \end{array}$

401 Note: Diagnostic Group was coded $0=$ typically developing, $1=$ autism spectrum disorder.

402 Gender was coded $0=$ female, $1=$ male.

403

404 
405 Table 3. Correlations per Diagnostic Group

$\begin{array}{lrrrrrr} & \text { Gender } & \text { Hours } & \text { FreeTime\% } & \text { Escapism } & \text { RawPGU } & \text { PGU } \\ \text { Gender } & - & .05 & .31 & .27 & .33 & .39 \\ \text { Daily game use (hrs) } & .28 & - & .51 & .06 & .18 & .25 \\ \text { Free time gaming (\%) } & .33 & .72 & - & .40 & .40 & .44 \\ \text { GAMES Escapism } & .42 & .42 & .48 & - & .34 & .40 \\ \text { PGU symptoms (raw) } & .27 & .62 & .69 & .42 & - & .94 \\ \text { PGU symptoms } & .34 & .67 & .66 & .49 & .94 & -\end{array}$

406

(transformed)

407 Note: Correlations calculated separately by group, with the ASD group displayed above the 408 diagonal and the TD group displayed below the diagonal. Gender was coded $0=$ female, $1=$ 409 male.

410 


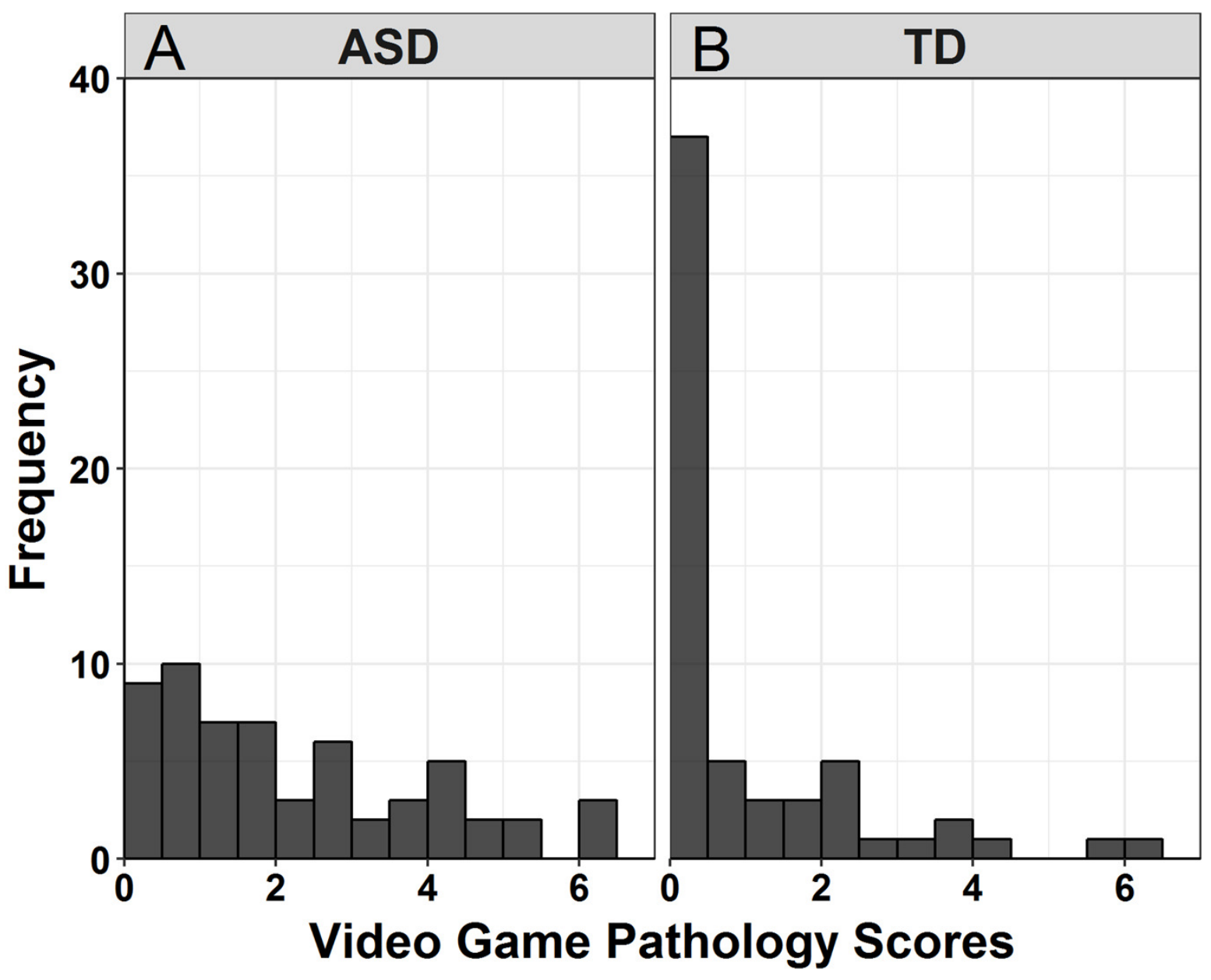

412 Figure 1. Histograms of pathological game use scores by diagnostic group. Figure 1A:

413 Histogram of scores in ASD group. Figure 1B: Histogram of scores in TD group. 

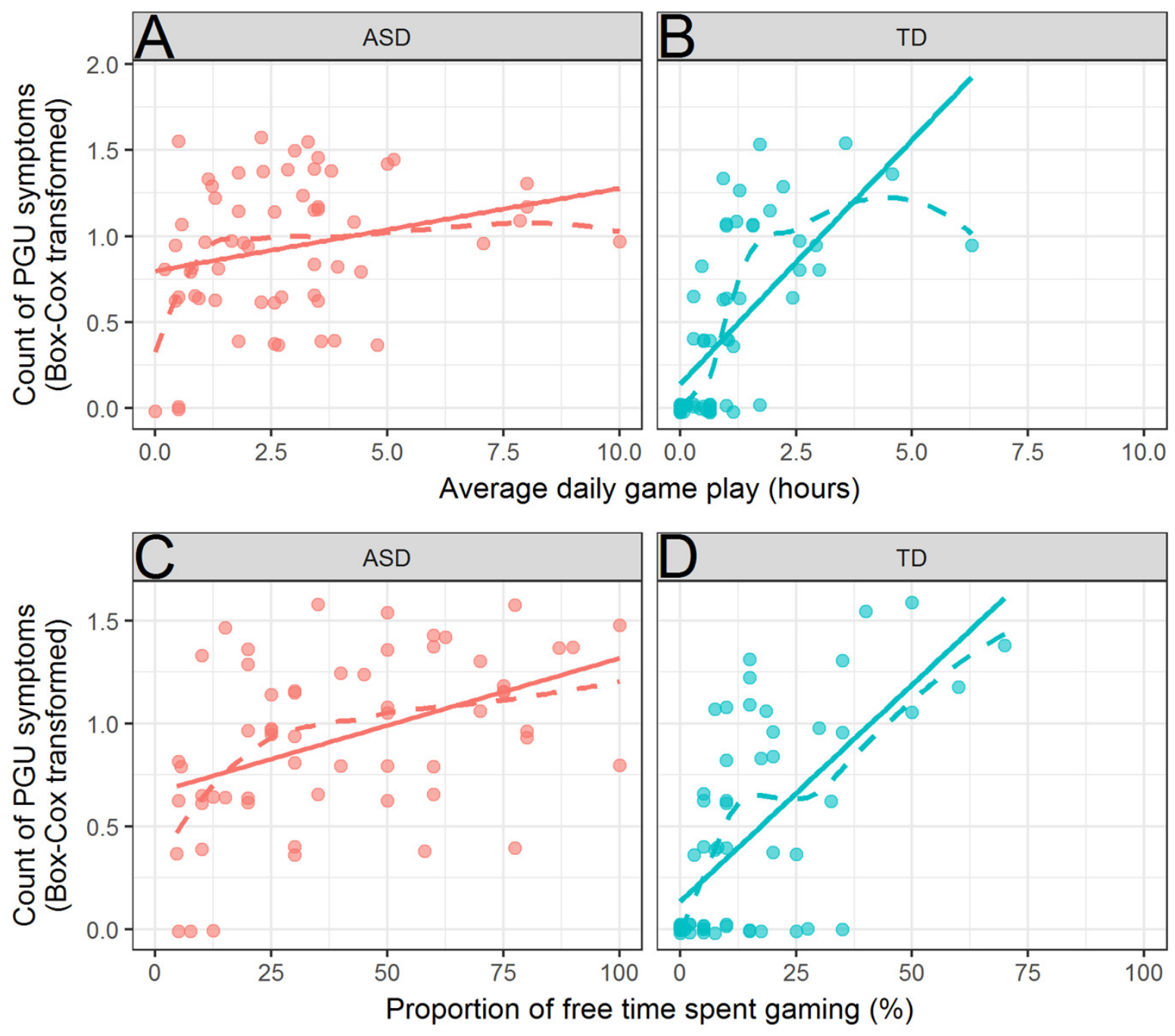

416

Figure 2. Relationships between time spent gaming (hours, Figure $2 \mathrm{~A}$ and $2 \mathrm{~B}$, and proportion, 418 Figure 2C and 2D) and PGU symptom count (Box-Cox transformed). Relationships are plotted 419 separately for ASD (Figure 2A and 2C) and TD (Figure 2B and 2D) groups. Slight vertical jitter 420 has been added to datapoints to reduce overplotting. Linear regression displayed in solid line, 421 locally weighted average displayed in dashed line. 
423

424

425

426

427

428

429

430

431

432

433

434

435

436

437

438

439

440

441

442

443

444

445

446

\section{References}

American Psychiatric Association. (2013). Diagnostic and Statistical Manual of Mental Disorders, Fifth Edition (DSM-5). Washington, DC: American Psychiatric Association.

Ben Shalom, D., Mostofsky, S., Hazlett, R., Goldberg, M., Landa, R., Faran, Y., . . Hoehn-Saric, R. (2006). Normal physiological emotions but differences in expression of conscious feelings in children with high-functioning autism. Journal of autism and developmental disorders, 36(3), 395-400. doi:10.1007/s10803-006-0077-2

Billstedt, E., Gillberg, C., \& Gillberg, C. (2005). Autism after adolescence: population-based 13-to 22-year follow-up study of 120 individuals with autism diagnosed in childhood. Journal of autism and developmental disorders, 35(3), 351-360. doi:10.1007/s10803-005-3302-5

Billstedt, E., Gillberg, I. C., \& Gillberg, C. (2007). Autism in adults: symptom patterns and early childhood predictors. Use of the DISCO in a community sample followed from childhood. Journal of Child Psychology and Psychiatry, 48(11), 1102-1110. doi:10.1111/j.1469-7610.2007.01774.x

Chuang, Y.-C. (2006). Massively Multiplayer Online Role-Playing Game-Induced Seizures: ANeglected Health Problem in Internet Addiction. CyberPsychology \& Behavior, 9(4), 451-456. doi:10.1089/cpb.2006.9.451

Dalton, K. M., Nacewicz, B. M., Johnstone, T., Schaefer, H. S., Gernsbacher, M. A., Goldsmith, H., .. . Davidson, R. J. (2005). Gaze fixation and the neural circuitry of face processing in autism. Nature neuroscience, 8(4), 519-526. doi:10.1038/nn1421

Durkin, K., Boyle, J., Hunter, S., \& Conti-Ramsden, G. (2015). Video games for children and adolescents with special educational needs. Zeitschrift für Psychologie. doi:10.1027/2151-2604/a000138

Eaves, L. C., \& Ho, H. H. (2008). Young adult outcome of autism spectrum disorders. Journal of autism and developmental disorders, 38(4), 739-747. doi:10.1007/s10803-007-0441-x 
447 Eickhoff, E., Yung, K., Davis, D. L., Bishop, F., Klam, W. P., \& Doan, A. P. (2015). Excessive video game use,

448

449

450

451

452

453

454

455

456

457

458

459

460

461

462

463

464

465

466

467

468

469

sleep deprivation, and poor work performance among US Marines treated in a military mental health clinic: a case series. Military medicine, 180(7), e839-e843. doi:10.7205/MILMED-D-1400597

Engelhardt, C. R., Mazurek, M. O., Hilgard, J., Rouder, J. N., \& Bartholow, B. D. (2015). Effects of violentvideo-game exposure on aggressive behavior, aggressive-thought accessibility, and aggressive affect among adults with and without autism spectrum disorder. Psychological science, 26(8), 1187-1200.

Engelhardt, C. R., Mazurek, M. O., \& Sohl, K. (2013). Media use and sleep among boys with autism spectrum disorder, ADHD, or typical development. Pediatrics, 132(6), 1081-1089. doi:10.1542/peds.2013-2066

Feng, Y., Yan, X., Guo, X., Wang, C., Li, Z., \& An, B. (2003). Behavior problem and family environment of children with video game dependence. Chinese Mental Health Journal, 17(6), 367-368.

Ferguson, B., Anderson-Hanley, C., Mazurek, M. O., Parsons, S., \& Warren, Z. (2012). Game interventions for autism spectrum disorder. GAMES FOR HEALTH: Research, Development, and Clinical Applications, 1(4), 248-253. doi:10.1089/g4h.2012.0717

Gentile, D. A. (2009). Pathological video-game use among youth ages 8 to 18 A National Study. Psychological science, 20(5), 594-602. doi:10.1111/j.1467-9280.2009.02340.x

Gentile, D. A., Choo, H., Liau, A., Sim, T., Li, D., Fung, D., \& Khoo, A. (2011). Pathological video game use among youths: a two-year longitudinal study. Pediatrics, peds. 2010-1353. doi:10.1542/peds.2010-1353

Gillott, A., \& Standen, P. (2007). Levels of anxiety and sources of stress in adults with autism. Journal of intellectual disabilities, 11(4), 359-370. doi:10.1177/1744629507083585 
470 Griffiths, M. D. (2008). Videogame addiction: Further thoughts and observations. International Journal of 471 Mental Health and Addiction, 6(2), 182-185. doi:10.1007/s11469-007-9128-y

472 Griffiths, M. D., Davies, M. N., \& Chappell, D. (2003). Breaking the stereotype: The case of online gaming. CyberPsychology \& Behavior, 6(1), 81-91. doi:10.1089/109493103321167992

474

475

476

477

478

479

480

481

482

483

484

485

486

487

488

489

490

491

492 reports, 82(2), 475-480. doi:10.2466/pr0.1998.82.2.475

Griffiths, M. D., Kuss, D. J., \& King, D. L. (2012). Video game addiction: Past, present and future. Current Psychiatry Reviews, 8(4), 308-318. doi:10.2174/157340012803520414

Grüsser, S. M., Thalemann, R., \& Griffiths, M. D. (2006). Excessive computer game playing: evidence for addiction and aggression? CyberPsychology \& Behavior, 10(2), 290-292. doi:10.1089/cpb.2006.9956

Grynszpan, O., Weiss, P. L. T., Perez-Diaz, F., \& Gal, E. (2014). Innovative technology-based interventions for autism spectrum disorders: A meta-analysis. Autism, 18(4), 346-361. doi:10.1177/1362361313476767

Hart, G. M., Johnson, B., Stamm, B., Angers, N., Robinson, A., Lally, T., \& Fagley, W. H. (2009). Effects of video games on adolescents and adults. CyberPsychology \& Behavior, 12(1), 63-65. doi:10.1089/cpb.2008.0117

Hellman, M., Schoenmakers, T. M., Nordstrom, B. R., \& van Holst, R. J. (2013). Is there such a thing as online video game addiction? A cross-disciplinary review. Addiction Research \& Theory, 21(2), 102-112. doi:10.3109/16066359.2012.693222

Hilgard, J., Engelhardt, C. R., \& Bartholow, B. D. (2013). Individual differences in motives, preferences, and pathology in video games: the gaming attitudes, motives, and experiences scales (GAMES). Frontiers in psychology, 4, 608. doi:10.3389/fpsyg.2013.00608 
493 Howlin, P., Goode, S., Hutton, J., \& Rutter, M. (2004). Adult outcome for children with autism. Journal of 494 Child Psychology and Psychiatry, 45(2), 212-229. doi:10.1111/j.1469-7610.2004.00215.x

495 Joseph, R. M., Ehrman, K., McNally, R., \& Keehn, B. (2008). Affective response to eye contact and face

496

497

498

499

500

501

502

503

504

505

506

507

508

509

510

511

512

513

514

515

516 recognition ability in children with ASD. Journal of the International Neuropsychological Society, 14(06), 947-955. doi:10.1017/S1355617708081344

Kelley, K. J., \& Gruber, E. M. (2012). Problematic Internet use and physical health. Journal of behavioral addictions, 2(2), 108-112. doi:10.1556/JBA.1.2012.016

King, D. L., Delfabbro, P. H., \& Zajac, I. T. (2011). Preliminary validation of a new clinical tool for identifying problem video game playing. International Journal of Mental Health and Addiction, 9(1), 72-87. doi:10.1007/s11469-009-9254-9

King, D. L., Haagsma, M. C., Delfabbro, P. H., Gradisar, M., \& Griffiths, M. D. (2013). Toward a consensus definition of pathological video-gaming: A systematic review of psychometric assessment tools. Clinical psychology review, 33(3), 331-342. doi:10.1016/j.cpr.2013.01.002

Lemmens, J. S., Valkenburg, P. M., \& Peter, J. (2009). Development and validation of a game addiction scale for adolescents. Media Psychology, 12(1), 77-95. doi:10.1080/15213260802669458

Lemmens, J. S., Valkenburg, P. M., \& Peter, J. (2011). Psychosocial causes and consequences of pathological gaming. Computers in Human Behavior, 27(1), 144-152. doi:10.1016/j.chb.2010.07.015

Levy, A., \& Perry, A. (2011). Outcomes in adolescents and adults with autism: A review of the literature. Research in Autism Spectrum Disorders, 5(4), 1271-1282. doi:10.1016/j.rasd.2011.01.023

Lord, C., DiLavorne, P. C., \& Risi, S. (2002). Autism Diagnostic Observation Schedule. Los Angeles, CA: Western Psychological Services.

Lord, C., Rutter, M., \& Le Couteur, A. (1994). Autism Diagnostic Interview-Revised: a revised version of a diagnostic interview for caregivers of individuals with possible pervasive developmental 
disorders. Journal of autism and developmental disorders, 24(5), 659-685. doi:10.1007/BF02172145

Losh, M., \& Capps, L. (2006). Understanding of emotional experience in autism: insights from the personal accounts of high-functioning children with autism. Developmental psychology, 42(5), 809. doi:10.1037/0012-1649.42.5.809

MacMullin, J. A., Lunsky, Y., \& Weiss, J. A. (2015). Plugged in: Electronics use in youth and young adults with autism spectrum disorder. Autism, 1362361314566047. doi:10.1177/1362361314566047

Mazefsky, C. A., Folstein, S. E., \& Lainhart, J. E. (2008). Overrepresentation of mood and anxiety disorders in adults with autism and their first-degree relatives: what does it mean? Autism Research, 1(3), 193-197. doi:10.1002/aur.23

Mazurek, M. O., \& Engelhardt, C. R. (2013a). Video game use and problem behaviors in boys with autism spectrum disorders. Research in Autism Spectrum Disorders, 7(2), 316-324.

Mazurek, M. O., \& Engelhardt, C. R. (2013b). Video game use in boys with autism spectrum disorder, ADHD, or typical development. Pediatrics, 132(2), 260-266. doi:10.1542/peds.2012-3956

Mazurek, M. O., Engelhardt, C. R., \& Clark, K. E. (2015). Video games from the perspective of adults with autism spectrum disorder. Computers in Human Behavior, 51, 122-130.

Mazurek, M. O., Shattuck, P. T., Wagner, M., \& Cooper, B. P. (2012). Prevalence and correlates of screenbased media use among youths with autism spectrum disorders. Journal of autism and 1258-1271. doi:10.1007/s10803-012-1659-9 
541 Mentzoni, R. A., Brunborg, G. S., Molde, H., Myrseth, H., Skouverøe, K. J. M., Hetland, J., \& Pallesen, S.

542 (2011). Problematic video game use: estimated prevalence and associations with mental and

543 physical health. Cyberpsychology, behavior, and social networking, 14(10), $591-596$.

544 doi:10.1089/cyber.2010.0260

545 Moore, D., \& Taylor, J. (2000). Interactive multimedia systems for students with autism. Journal of

$546 \quad$ Educational Media, 25(3), 169-177. doi:10.1080/1358165000250302

547 Morey, R., Rouder, J., \& Jamil, T. (2014). BayesFactor: Computation of Bayes factors for common

$548 \quad$ designs. $R$ package version $0.9,8$.

549 Moseley, D. S., Tonge, B. J., Brereton, A. V., \& Einfeld, S. L. (2011). Psychiatric comorbidity in adolescents

550 and young adults with autism. Journal of Mental Health Research in Intellectual Disabilities, 4(4),

$551 \quad$ 229-243. doi:10.1080/19315864.2011.595535

552 Müller, E., Schuler, A., \& Yates, G. B. (2008). Social challenges and supports from the perspective of

553 individuals with Asperger syndrome and other autism spectrum disabilities. Autism, 12(2), 173-

554 190. doi:10.1177/1362361307086664

555 Orsmond, G. I., \& Kuo, H.-Y. (2011). The daily lives of adolescents with an autism spectrum disorder

556 Discretionary time use and activity partners. Autism, 15(5), 579-599.

557 doi:10.1177/1362361310386503

558

559

Orsmond, G. I., Shattuck, P. T., Cooper, B. P., Sterzing, P. R., \& Anderson, K. A. (2013). Social participation .

560 among young adults with an autism spectrum disorder. Journal of autism and developmental

561 disorders, 43(11), 2710-2719. doi:10.1007/s10803-013-1833-8

Petry, N. M., Rehbein, F., Gentile, D. A., Lemmens, J. S., Rumpf, H. J., Mößle, T., . . Borges, G. (2014). An 562 international consensus for assessing internet gaming disorder using the new DSM-5 approach. Addiction, 109(9), 1399-1406. doi:10.1111/add.12457 
564 Scharkow, M., Festl, R., \& Quandt, T. (2014). Longitudinal patterns of problematic computer game use

565 among adolescents and adults - a 2-year panel study. Addiction, 109(11), 1910-1917.

566 doi:10.1111/add.12662

567 Seltzer, M. M., Shattuck, P., Abbeduto, L., \& Greenberg, J. S. (2004). Trajectory of development in

568 adolescents and adults with autism. Mental retardation and developmental disabilities research

569 reviews, $10(4), 234-247$. doi:10.1002/mrdd.20038

570 Shattuck, P. T., Seltzer, M. M., Greenberg, J. S., Orsmond, G. I., Bolt, D., Kring, S., ... Lord, C. (2007).

$571 \quad$ Change in autism symptoms and maladaptive behaviors in adolescents and adults with an

572 autism spectrum disorder. Journal of autism and developmental disorders, 37(9), $1735-1747$.

573 doi:10.1007/s10803-006-0307-7

574 Sim, T., Gentile, D. A., Bricolo, F., Serpelloni, G., \& Gulamoydeen, F. (2012). A conceptual review of

575 research on the pathological use of computers, video games, and the Internet. International

576 Journal of Mental Health and Addiction, 10(5), 748-769. doi:10.1007/s11469-011-9369-7

577 Smyth, J. M. (2007). Beyond self-selection in video game play: an experimental examination of the

578 consequences of massively multiplayer online role-playing game play. CyberPsychology \&

579 Behavior, 10(5), 717-721. doi:10.1089/cpb.2007.9963

580 Taylor, J. L., \& Seltzer, M. M. (2010). Changes in the autism behavioral phenotype during the transition

581 to adulthood. Journal of autism and developmental disorders, 40(12), 1431-1446.

582 doi:10.1007/s10803-010-1005-z

583 Tejeiro Salguero, R. A., \& Morán, R. M. B. (2002). Measuring problem video game playing in adolescents.

$584 \quad$ Addiction, 97(12), 1601-1606. doi:10.1046/j.1360-0443.2002.00218.x

585 